Supporting Information for

\title{
Aluminum-Based Surface Polymerization on Carbon Dots with Aggregation- Enhanced Luminescence
}

Yunyang Zhao a, Bingchen He a, Enshan Liu a , Jielei Li a, Liming Wang a, Shi Chen a, Yeqing Chen

b, Zhan'ao Tan ${ }^{\mathrm{c}}$, Kar Wei Nga,*, Shuangpeng Wang a,*, Zikang Tang a and Songnan Qu ${ }^{\mathrm{a}, *}$

a Joint Key Laboratory of the Ministry of Education, Institute of Applied Physics and Materials Engineering, University of Macau, Taipa 999078, Macau SAR, P. R. China.

${ }^{\mathrm{b}}$ School of Applied Physics and Materials, Wuyi University, Jiangmen, Guangdong, 529020, P. R. China.

${ }^{\mathrm{c}}$ Beijing Advanced Innovation Center for Soft Matter Science and Engineering, Beijing University of Chemical Technology, Beijing 100029, P. R. China

* Corresponding author.

Email: (S.Q.) songnanqu@um.edu.mo, (S.W.) spwang@um.edu.mo, (K.W.N.) billyng@um.edu.mo. 


\section{Experiment section}

Synthesis of Na-CDs, Ca-CDs and Al-CDs

The Na-CDs, Ca-CDs and Al-CDs powder were obtained by one-step microwave synthesis by adding $1.105 \mathrm{~g}$ of $\mathrm{NaCl}$ (containing $0.434 \mathrm{~g} \mathrm{Na}$ ), $1.105 \mathrm{~g}$ of $\mathrm{CaCl}_{2}$ (containing $0.398 \mathrm{~g} \mathrm{Ca}$ ) or $2 \mathrm{~g}$ of $\mathrm{AlCl}_{3} \cdot 6 \mathrm{H}_{2} \mathrm{O}$ (containing $0.223 \mathrm{~g} \mathrm{Al}$, equivalent to $1.105 \mathrm{~g}$ anhydrous $\mathrm{AlCl}_{3}, 99.9 \% \mathrm{AR}$, Aladdin, China. Do not use anhydrous $\mathrm{AlCl}_{3}$ directly), respectively, with $1.5 \mathrm{~g}$ of citric acid $(99.5 \% \mathrm{AR}$, Aladdin, China), $3 \mathrm{~g}$ of urea $(99.5 \% \mathrm{AR}$, Aladdin, China) in $10 \mathrm{~mL}$ ultrapure water $(18.25 \mathrm{M} \Omega \cdot \mathrm{cm})$ to form transparent colorless solutions in beakers. Then the solutions were heated in a domestic microwave oven separately. After $\sim 3$ minutes with the water being evaporated, the solutions were turned into carbon dots powder with $\mathrm{Na}, \mathrm{Ca}$, and $\mathrm{Al}$, named Na-CDs, Ca-CDs and Al-CDs, respectively. CAUTION! During the synthesis process, do not dissolve the anhydrous $\mathrm{AlCl}_{3}$ in water for precursor solution because anhydrous $\mathrm{AlCl}_{3}$ will hydrolyze violently in water. All the experiment process should be operated in a fume hood, especially the microwave synthesis process because some pungent volatile gases came out during the microwave synthesis.

\section{Characterization}

The UV vis absorption spectra were measured on a UV-2600 spectrophotometer (Shimadzu Corporation, Japan). PL, PLE and EEM spectra were recorded on an FS5 fluorescence spectrophotometer (Edinburgh instruments Ltd., UK) by using a Xe lamp as an excitation source. Fourier transform infrared (FTIR) spectra tested on TENSOR II FTIR Spectrometer (Bruker Optics, Germany). The TEM images were acquired by Talos F200X Transmission Electron Microscope (FEI, USA) operating at $200 \mathrm{kV}$. The CDs samples were prepared by being dissolved in water, then dropped 
on a coper grid with ultrathin carbon film and dried under low pressure. The AFM test was employed on a BRUKER ICON Atomic Force Microscope. The samples were prepared by dropping the CDs aqueous solution onto the smooth mica and dried under low pressure. The absolute PLQY was measured by Edinburgh FS5 fluorescence spectrophotometer with SC-30 integrating sphere holder using an instrument supplied plug as reference sample. The PLQY equals to the ratio of integral area of emission and scattering spectrum between CDs sample and reference sample. Time-resolved PL decay curves were tested by the technique of Time Correlated Single Photon Counting (TCSPC) on the FS5 fluorescence spectrophotometer with a ps pulsed laser of $375 \mathrm{~nm}$. XPS spectra were carried out on ESCALAB 250Xi spectrometer (Thermo Fisher Scientific, United States) with a monochromatic $\mathrm{X}$-ray source $(\mathrm{Al} \mathrm{K} \alpha, \mathrm{h} v=1486.7 \mathrm{eV})$ in the ultrahigh vacuum chamber under the base pressure of $1.0^{*} 10^{-10}$ mbar. The flood gun was opened to give the charge compensation. The total energy resolution was determined to be $0.3 \mathrm{eV}$.

\section{Photostability of Al-CDs, $r$-CDs and commercial organic dye}

For Al-CDs, the PL spectra of Al-CDs powder excited at $420 \mathrm{~nm}$ was directly tested for each 1015min under $365 \mathrm{~nm}$ UV light irradiation. For r-CDs and C545T dye (a commercial organic dye), $0.2 \mathrm{mg} / \mathrm{mL}$ of their aqueous solution dropped onto filter papers and dried in an oven. Then the filter papers with r-CDs and dye were used for PL measurement (excited at $420 \mathrm{~nm}$ ). During the interval time between two repeated PL measurements, the Al-CDs powder, r-CDs and organic dye coated filter paper were irradiated under $365 \mathrm{~nm}$ UV light from $150 \mathrm{~W}$ Xe lamp in the FS5 fluorescence spectrophotometer of Edinburgh instruments. The PL peak intensity values were extracted from PL spectra at different irradiation time to plot the photostability curves. 
Fabrication of Al-CDs based white LED

The Al-CDs powder was used as a phosphor to coat onto an InGaN based LED chip to fabricate the white LED. The emission wavelength of InGaN based LED chip was centered at $450 \mathrm{~nm}$ under the working voltage of $3.0 \mathrm{~V}$. In detail, $50 \mathrm{mg}$ of Al-CDs powder was dispersed into $450 \mu \mathrm{L}$ of $195 \mathrm{~T}$ potting glue (A) and $45 \mu \mathrm{L}$ of curing agent (B) (Shenzhen Ausbond Co.,LTD, China) and stirred with a needle to form a little viscous homogeneous solution. After that we dredged out a drop of this solution and dropped it onto the LED chip. Then the LED chip coated with Al-CDs was transferred into an oven and heated at $70{ }^{\circ} \mathrm{C}$ for $1 \mathrm{~h}$. At last, the white LED device was obtained. 

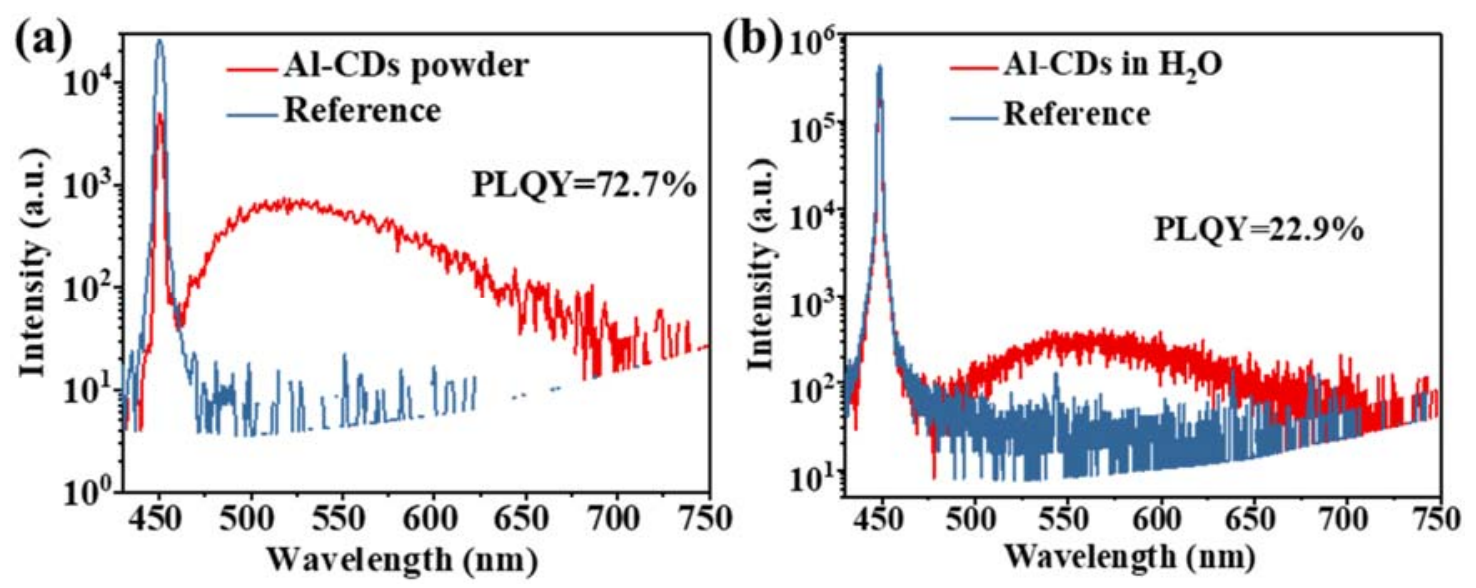

Figure S1. The PLQY of Al-CDs powder (a) and aqueous solution (b) excited at $450 \mathrm{~nm}$. The high PLQY of Al-CDs powder could be due to: (1) the aggregating of the green emissive carbon cores was avoided by considerable steric hindrance of the Al-based crosslinked polymerization on the surface of the CDs; (2) efficient ET from the blue emissive polymerized surface to the green emissive carbon cores. The low PLQY of Al-CDs aqueous solution could be due to the nonradiative charge recombination caused by water molecules. 

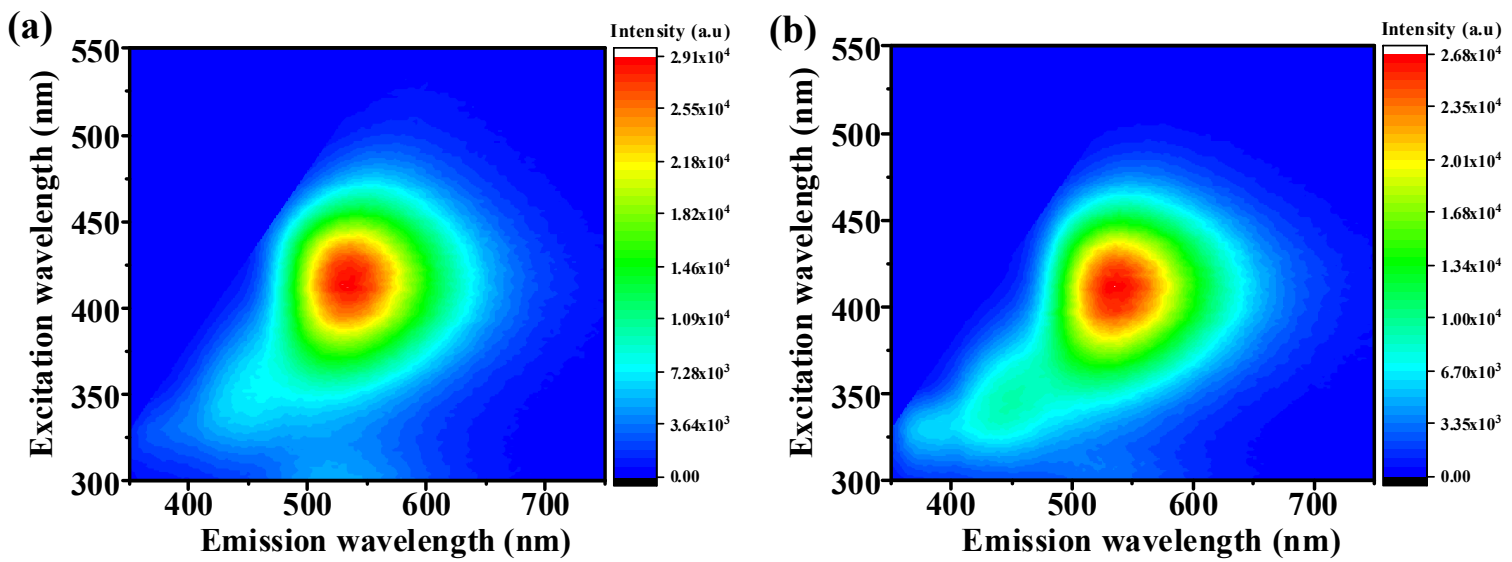

Figure S2. The EEM of (a) Na-CDs and (b) Ca-CDs aqueous solution. 


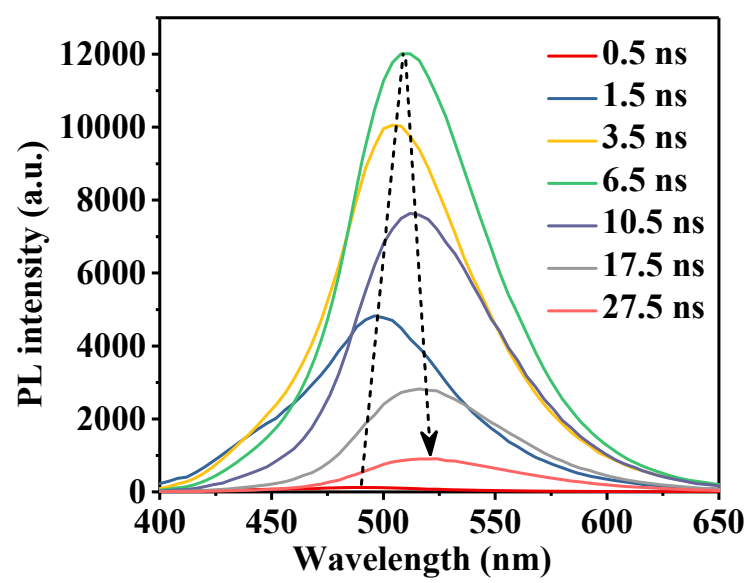

Figure S3. The sliced TRES of Al-CDs at different decay times tested with a ps pulsed laser of 375 nm. 

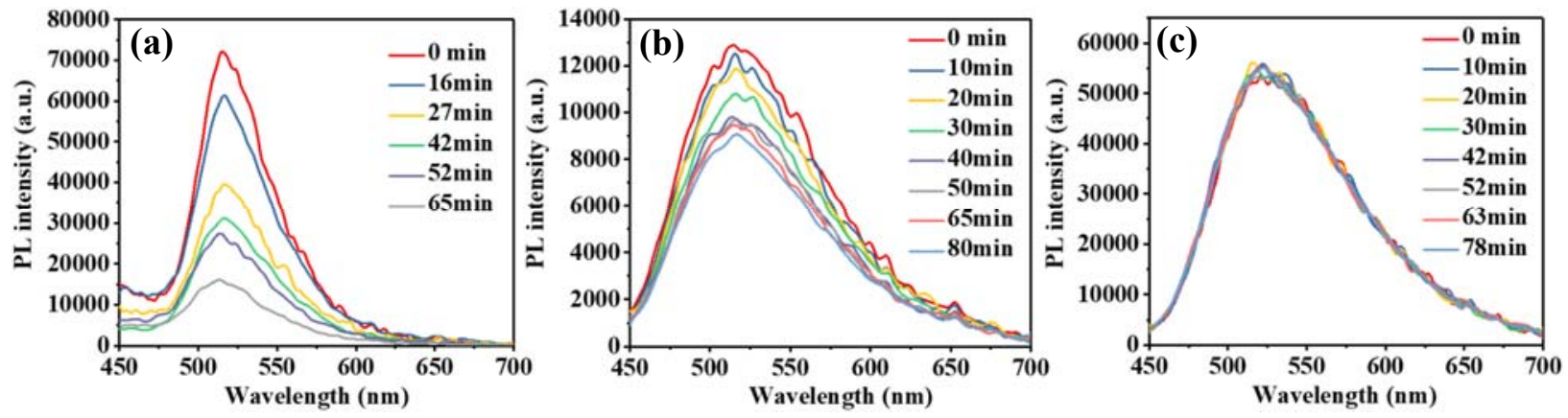

Figure S4. The PL spectra of commercial organic dye coated filter paper (a), r-CDs coated filter paper (b) and Al-CDs powder(c) irradiated continuously under different times. 
By utilizing the Al-CDs powder as a phosphor, down-conversion white LEDs (WLEDs) with Commission Internationale de l'Eclairage (CIE) chromaticity coordinates of $(0.35,0.33)$ with color temperature (CT) of $4600 \mathrm{~K}$ could be easily fabricated by depositing the Al-CDs powder on an InGaN LED chip (Figure S5). The Lumen efficiency $(\eta)$ and color rendering index (CRI) of the WLED were $25.6 \mathrm{~lm} / \mathrm{W}$ and 81.1 , respectively.
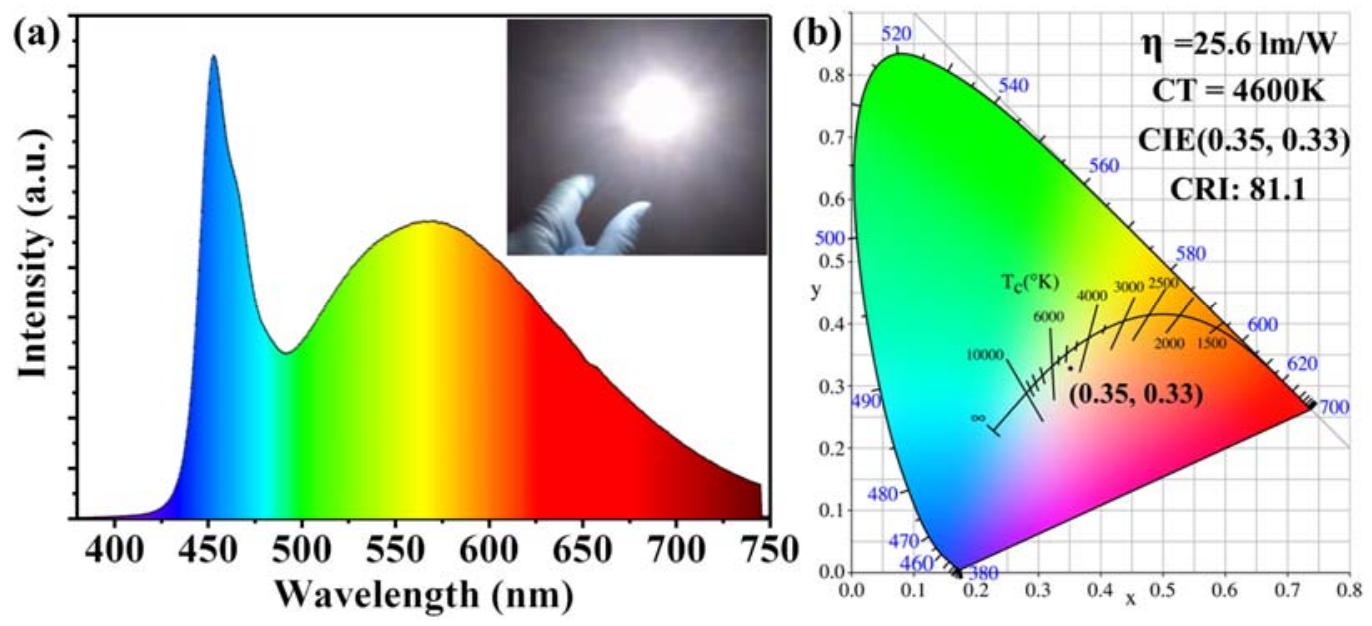

Figure S5. (a) The emission spectrum of Al-CDs powder based white LED; Insert shows the photo of this working LED prototype; (b) The CIE coordinate of the white LED and the corresponding parameters. 
The FTIR spectra in Figure S7(a) showed that the peak located at $1625 \mathrm{~cm}^{-1}$ and $\sim 1400 \mathrm{~cm}^{-1}$ corresponding to the stretching vibration of $-\mathrm{C}=\mathrm{O}$ and $\mathrm{C}-\mathrm{N}$ in amide groups, respectively. Absorption bands at $3350-3100 \mathrm{~cm}^{-1}$ and $1740-1700 \mathrm{~cm}^{-1}$ are assigned to $v(\mathrm{~N}-\mathrm{H})$ and $v(\mathrm{C}=\mathrm{O})$ in carboxyl group, respectively. ${ }^{1-2}$ Compared with $\mathrm{r}-\mathrm{CD}$, it can be found that Al-CDs have a higher contents of $\mathrm{N}$ containing groups like amide group in relative to carboxyl group, indicating the introduction of $\mathrm{Al}$ ions could suppress the $\mathrm{N}$ atom loss by forming coordination bonds with amide groups during the microwave heating process. ${ }^{3-4}$ Figure S7(b) exhibits the XPS survey spectra and the corresponding elemental contents of r-CDs, Na-CDs, Ca-CDs and Al-CDs listed in Table 1. During the microwave heating, large amount of urea was evaporated and decomposed into $\mathrm{NH}_{3}$ and $\mathrm{CO}_{2}$ leading to the lowest content $(8.48 \%)$ of $\mathrm{N}$ in $\mathrm{r}-\mathrm{CD}$. However, with different salt additives, the $\mathrm{N}$ content increases for 2-3 times in Na-CDs, Ca-CDs and Al-CDs than that in r-CDs. The ratios of $\mathrm{Cl}: \mathrm{Na}$ and $\mathrm{Cl}: \mathrm{Ca}$ in $\mathrm{Na}-\mathrm{CDs}$ and $\mathrm{Ca}-\mathrm{CDs}$ were close to the value in $\mathrm{NaCl}$ and $\mathrm{CaCl}_{2}$, respectively (Table $\mathrm{S} 1$ ). In contrast, the ratio of $\mathrm{Cl}: \mathrm{Al}$ in $\mathrm{Al}-\mathrm{CDs}$ is 0.33 which is far lower than that in $\mathrm{AlCl}_{3}$. 

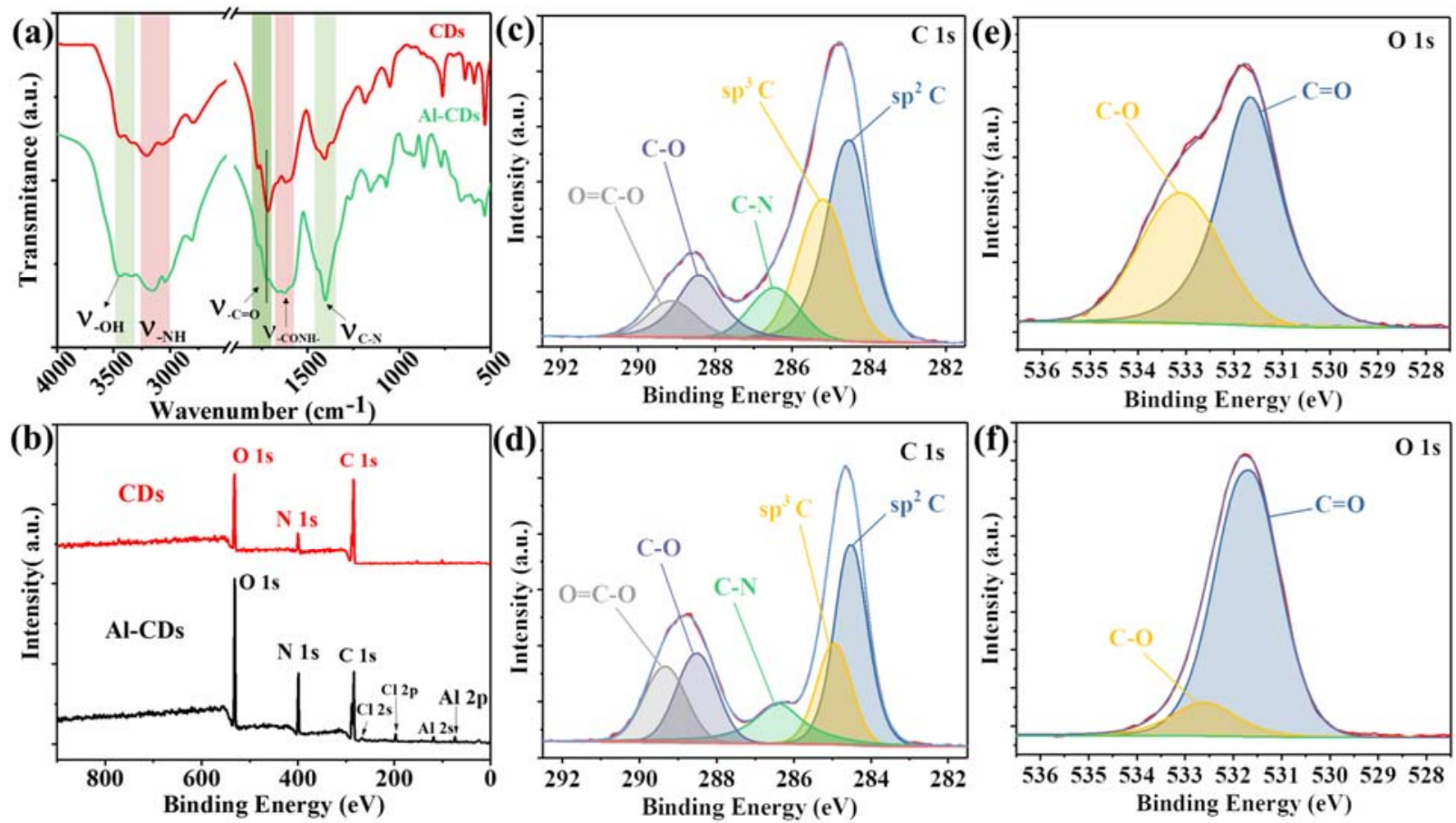

Figure S6. The FTIR (a) and XPS (b) spectra of r-CDs and Al-CDs; The high resolution XPS of C 1s, O 1s spectra of r-CDs (c, e) and Al-CDs (d, f), respectively. 

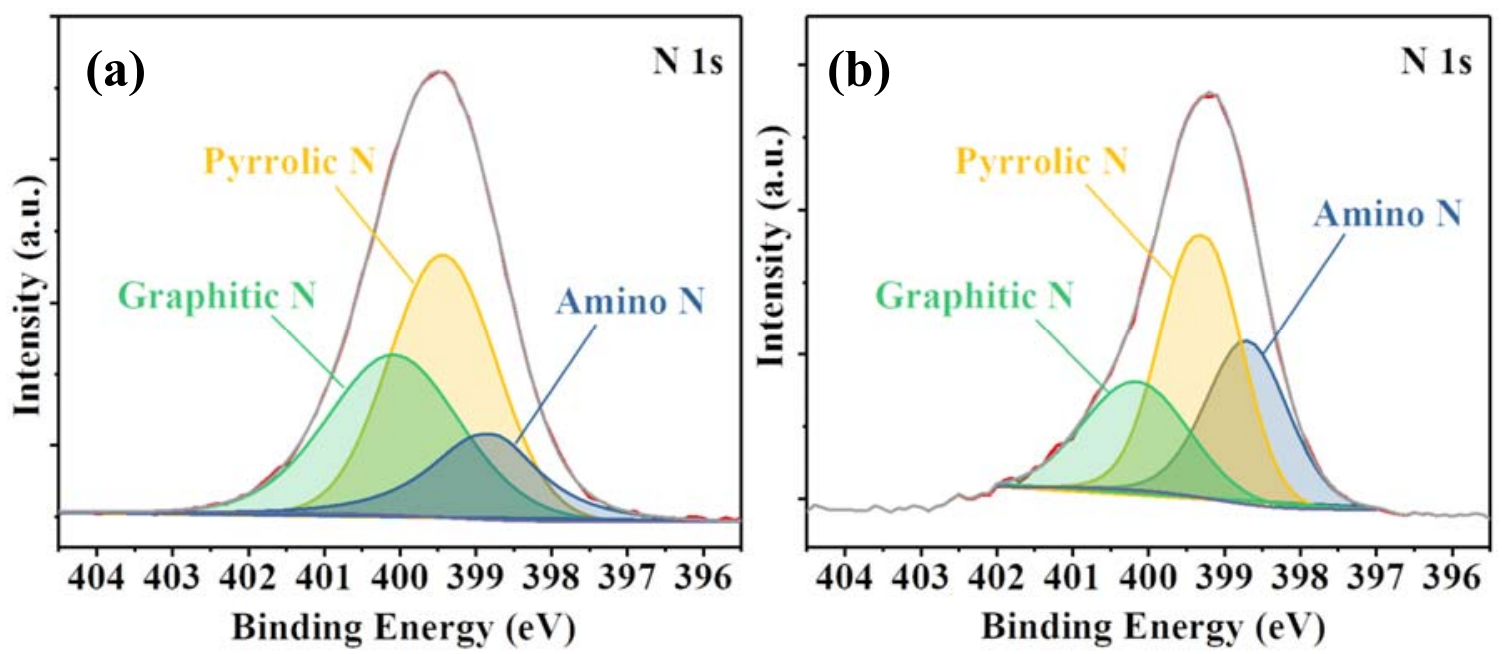

Figure S7. The high resolution XPS of N 1s spectra of (a) Na-CDs and (b) Ca-CDs, respectively. 
Table S1. The element contents of r-CDs, Na-CDs, Ca-CDs and Al-CDs from the XPS spectra.

\begin{tabular}{ccccccc}
\hline Samples & C, at.\% & N, at.\% & O, at.\% & $\mathrm{M}^{*}$, at.\% & Cl, at.\% & Cl/M*, at. \\
\hline r-CDs & 67.73 & 8.48 & 23.79 & - & - & - \\
Na-CDs & 44.68 & 25.68 & 24.33 & 2.69 & 2.63 & 0.98 \\
Ca-CDs & 33.2 & 22.91 & 35.2 & 3.02 & 5.66 & 1.87 \\
Al-CDs & 47.47 & 18.34 & 28.59 & 4.2 & 1.4 & 0.33 \\
\hline
\end{tabular}

* M stands for $\mathrm{Na}, \mathrm{Ca}$ and $\mathrm{Al}$ in $\mathrm{Na}-\mathrm{CDs}, \mathrm{Ca}-\mathrm{CDs}$ and $\mathrm{Al}-\mathrm{CDs}$, respectively. 


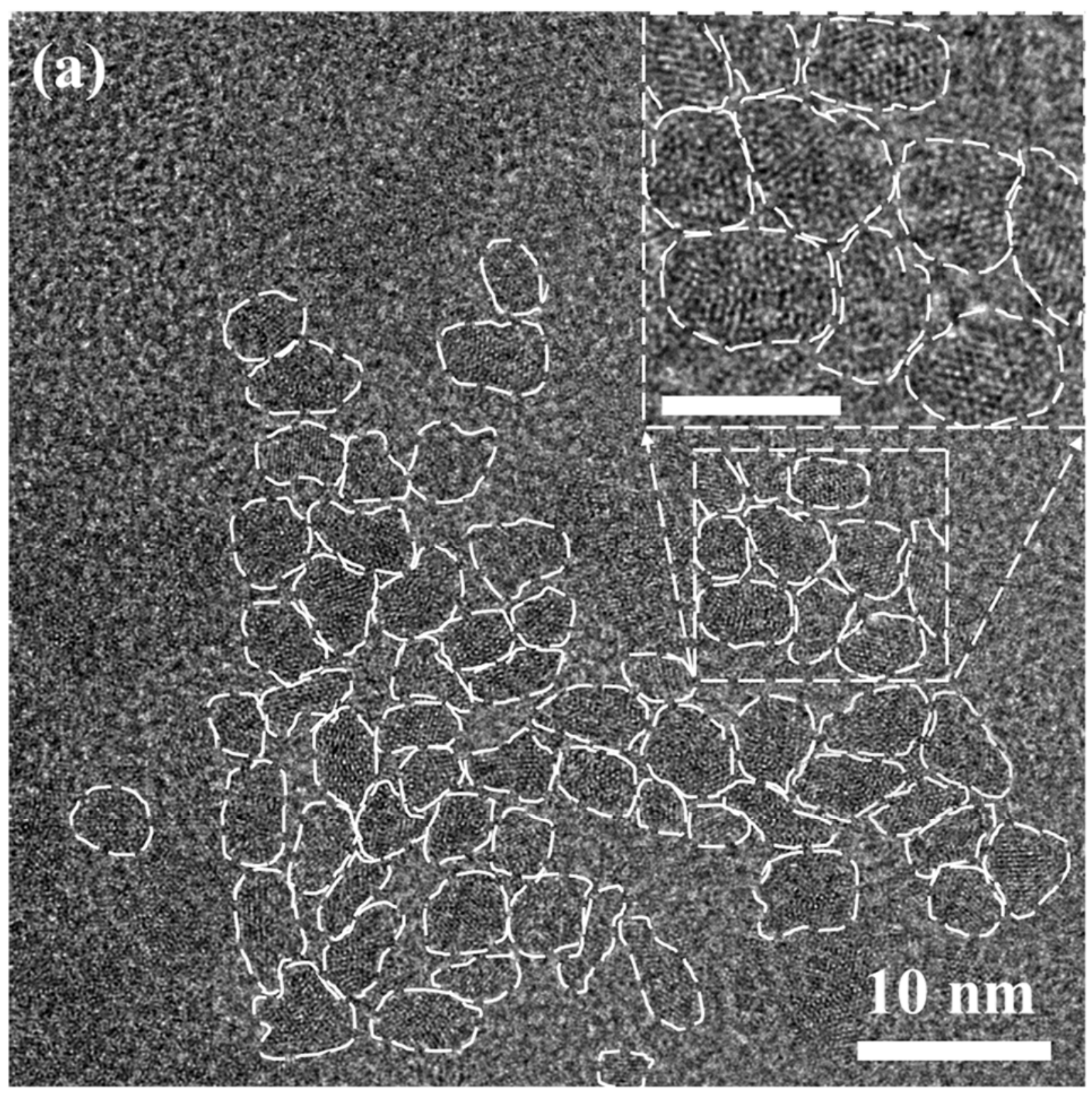

Figure S8. The TEM image of r-CDs (enlarged image from Figure 3a) 


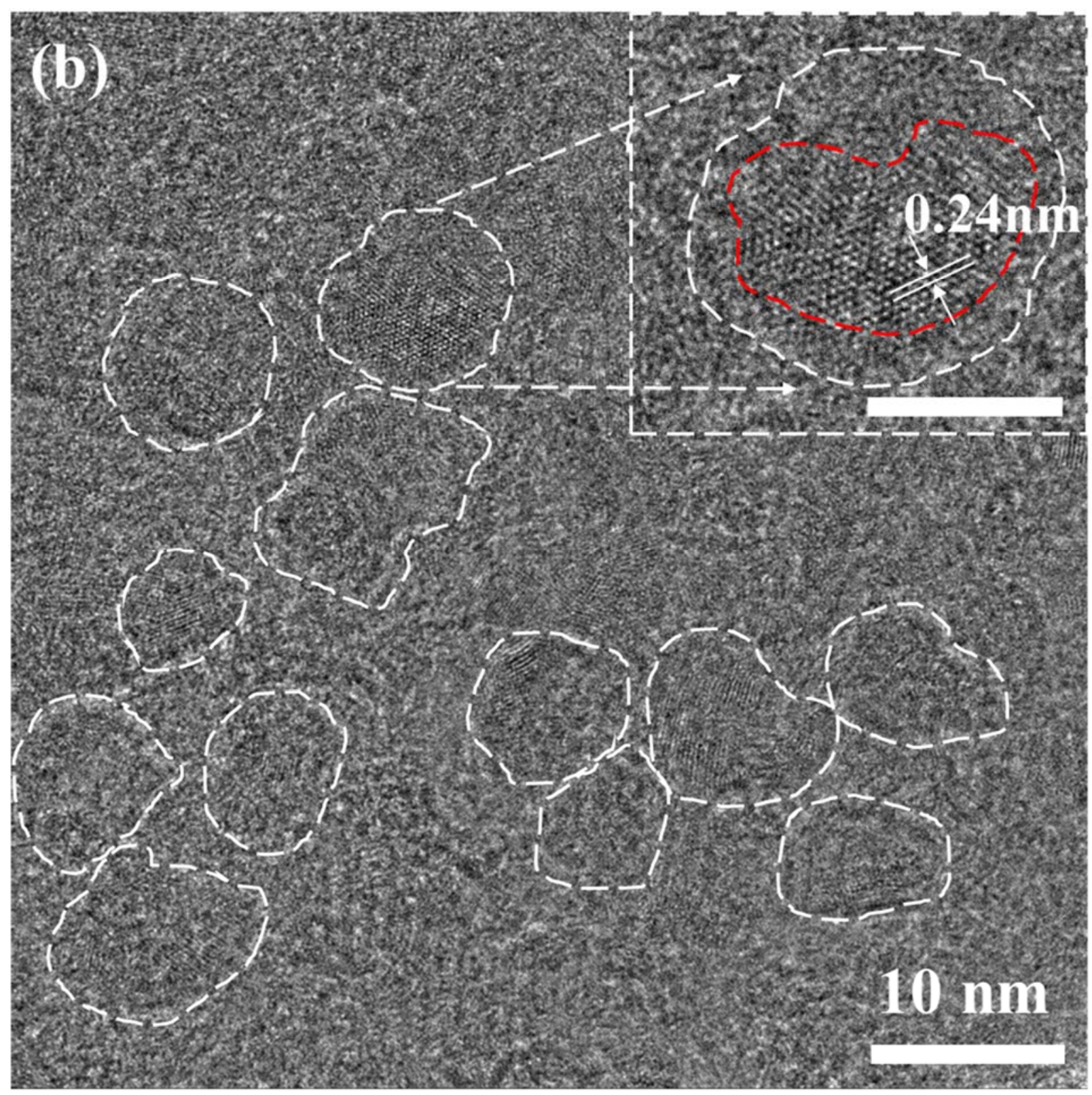

Figure S9. The TEM image of Al-CDs (enlarged image from Figure 3b) 


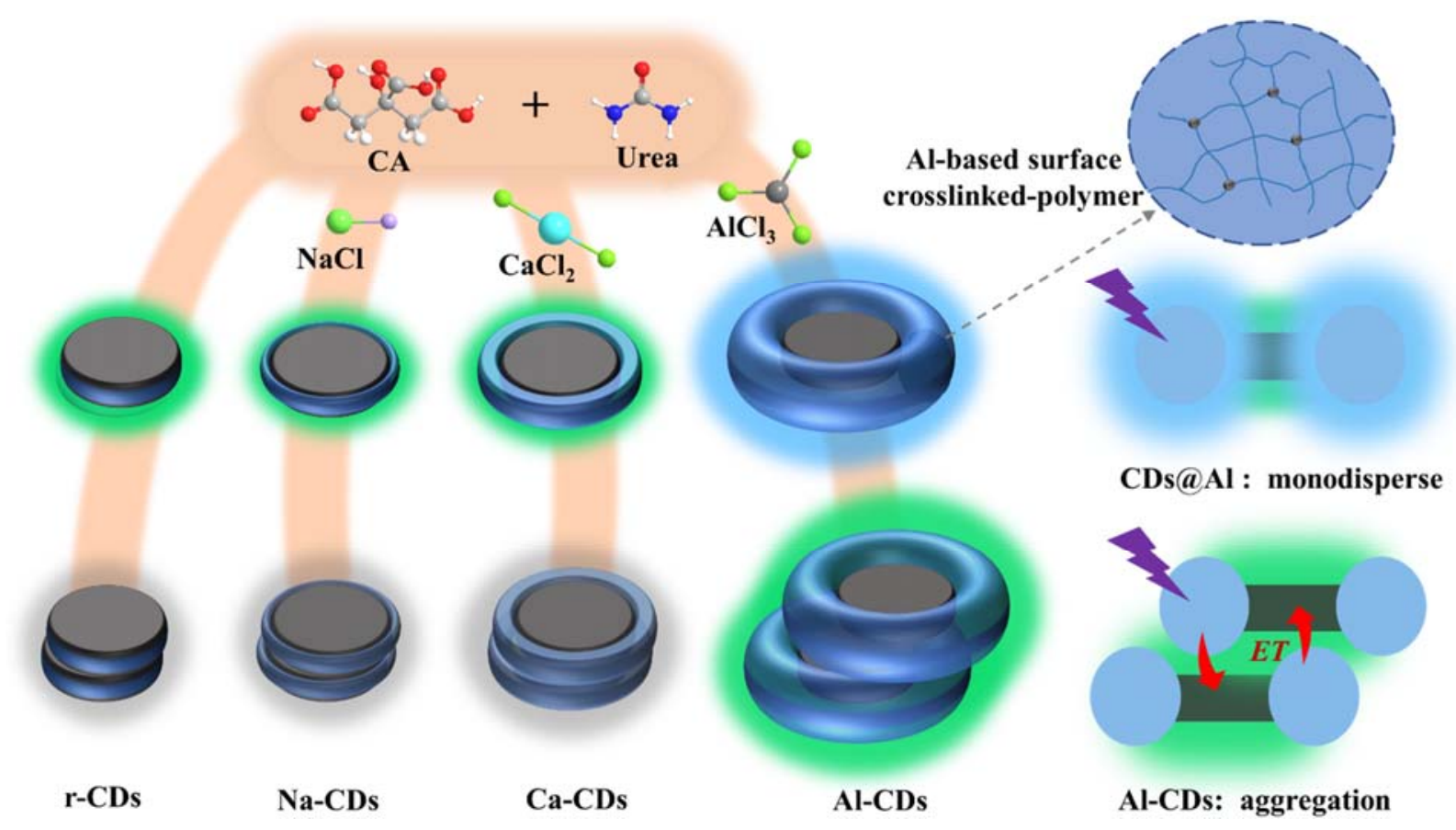

Schematic S1. The formation processes of r-CDs, Na-CDs, Ca-CDs and Al-CDs and the corresponding luminescent processes of $\mathrm{Al}-\mathrm{CDs}$ in monodisperse and aggregate. 


\section{References}

1. $\quad$ Feng, X. Y.; Ng, K. W.; Wang, S. P.; Chen, W. Z.; Zhang, Z. Z.; Chen, W.; Zhao, Y. Y.; Tu, B.; Tang, Z. K.; Pan, H.; $\mathrm{He}, \mathrm{Z}$. B. Investigation on the role of amines in the liquefaction and recrystallization process of $\mathrm{MAPbI}_{3}$ perovskite. Journal of Materials Chemistry A 2020, 8 (27), 13585-13593.

2. Miao, M.; Zuo, S.; Zhao, Y.; Wang, Y.; Xia, H.; Tan, C.; Gao, H. Selective oxidation rapidly decomposes biomassbased activated carbons into graphite-like crystallites. Carbon 2018, 140, 504-507.

3. Wang, R.; Xue, J.; Wang, K.-L.; Wang, Z.-K.; Luo, Y.; Fenning, D.; Xu, G.; Nuryyeva, S.; Huang, T.; Zhao, Y.; Yang, J. L.; Zhu, J.; Wang, M.; Tan, S.; Yavuz, I.; Houk, K. N.; Yang, Y. Constructive molecular configurations for surface-defect passivation of perovskite photovoltaics. Science 2019, 366 (6472), 1509-1513.

4. Wang, Q.; Yu, S.; Qin, W.; Wu, X. Isopropanol-assisted synthesis of highly stable $\mathrm{MAPbBr}_{3} / \mathrm{p}-\mathrm{g}-\mathrm{C}_{3} \mathrm{~N}_{4}$ intergrowth composite photocatalysts and their interfacial charge carrier dynamics. Nanoscale Advances 2020, 2 (1), 274-285. 